\title{
OMAE2009-79099
}

\section{DRAG COEFFICIENTS OF A LONG FLEXIBLE CIRCULAR CYLINDER WITH WAKE INTERFERENCE}

\author{
Francisco J Huera-Huarte \\ Graduate Aerospace Laboratories \\ California Institute of Technology \\ Pasadena, California 91125 \\ Email: fhuera@caltech.edu
}

\begin{abstract}
Drag coefficients of the downstream cylinder in a tandem arrangement of two models aligned with the flow are shown in this work. The models, which experienced vortex-induced vibrations with wake interference, had an external diameter of $16 \mathrm{~mm}$ and a total length of $1.5 \mathrm{~m}$ giving an aspect ratio of about 94. More than 400 runs were carried out in a water flume with only the lower $40 \%$ of the models exposed to the current. The flow speed was varied up to $0.75 \mathrm{~m} / \mathrm{s}$ giving Reynolds numbers in the range from 1200 to 12000. A supporting structure, where the models were attached, allowed changes in the centre to centre distances and in the applied top tensions. Separations of up to 4 diameters were tested with tension variations from 15 to $110 \mathrm{~N}$. Reduced velocities based on the fundamental natural frequency reached values up to 16. The mass ratio of the models was around 1.8 and the combined mass-damping parameter about 0.05 .
\end{abstract}

\section{NOMENCLATURE}

$C_{d}$ Drag coefficient

$c_{x}$ In-line curvature

$c_{y}$ Cross-flow curvature

$D$ External diameter

$E A$ Axial Stiffness

EI Flexural Stiffness

$E I^{*} \quad$ Modified flexural stiffness

$f_{1} \quad$ Fundamental natural frequency in water

$f_{x}$ In-line force distribution

$F_{X} \quad$ Total in-line force
$L \quad$ Length

$L / D$ Aspect ratio

$L_{s} \quad$ Submerged Length

$m$ Mass

$m^{*} \quad$ Mass ratio

$\left(m^{*}+C_{A}\right) \zeta$ Combined mass-damping parameter

Re Reynolds number

$S$ Centre to centre distance

$T_{t}$ Top Tension

$V$ Flow speed

$V_{1} \quad$ Reduced velocity based on $f_{1}$

$w_{s}$ Submerged weight

$W$ Point load at mid point

$x$ In-line displacements

$x_{M} \quad$ In-line deflection at mid point

$y$ Cross-flow displacements

$\rho$ Water density

$\zeta$ Damping ratio

\section{INTRODUCTION}

Knowledge about the hydrodynamic forces acting on flexible circular cylinders responding to vortex-induced vibrations (VIV) is fundamental for the design of offshore structures. Insitu measurements are always difficult and instrumentation is most of the times not designed to resolve completely the response of such structures. Most of the field data comes from accelerometers and hydrodynamic forcing is rarely reported. In contrast, 
some prediction tools used by the industry are based on measurements and hence, they rely on the quality of the measurements available. Even though excellent reviews on VIV have been reported [1], [2], [3], [4], [5] and [6], data showing drag forces on flexible cylinders is scarce and even more when there is wake interference between several bluff bodies.

A recent benchmarking exercise [7] in which several CFD and empirical prediction tools were used to simulate the response of a single long flexible cylinder, reported significant differences between the predicted response and that measured in laboratory experiments [8]. There is still the need for data showing force coefficients of different arrangements of flexible circular cylinders. The drag coefficients exhibited by long flexible cylinders are considerably larger than those typical of stationary or flexibly mounted rigid cylinders and this large values of drag coefficient are related to large amplitude responses.

Large values of drag coefficient were reported in towing experiments with long flexible cylinders and related to large amplitude motions in [9]. In another experiment with a very high aspect ratio cylinder [10], drag coefficients between 1.5 and 2.7 depending on the response experienced by the model were also reported. Similar values to those reported in those two studies, were observed in recent experimental work carried out in a large laboratory facility [8], where it was shown how the drag coefficients increased monotonically with values between 1 and 2.8, inside clearly defined mode related lock-in branches.

Offshore flexible structures such as marine deep water risers and mooring lines are more often found arranged in groups than solitary. In contrast, most of the studies in the past years have been focused into solitary flexibly mounted rigid or flexible circular cylinders. There is no data published showing drag coefficients of long flexible cylinders in tandem or other arrangements of several bodies when vibrating at high mode numbers, and this situation is of especial interest because of its practical implications in offshore design. The design of models and the facilities needed to accomplish multi-mode vortex-induced vibration experiments with wake interference in tandem or other arrangements, is certainly challenging. Keeping realistic mechanical characteristics in relatively small facilities is very difficult. Resolving high mode motion requires a large number of sensors and without it, the information one can obtain from the structure is very limited. Measuring hydrodynamic forces is also very challenging and this is the reason why the data available is very limited.

Vortex-induced vibrations, galloping and combinations of both types of response were observed in experiments [11] with a flexibly mounted rigid cylinder immersed in the wake of an stationary one with the same dimensions. Different configurations of side-by-side, staggered and tandem arrangements of flexible cantilevers of circular cross section have also been studied in a wind tunnel [12]. In the recent years, several authors have performed experiments to study vortex and wake induced vibrations of tandem arrangements of rigid cylinders [13] and [14].

In this work, two long flexible circular cylinders have been used and the drag coefficients acting on the trailing one, with wake interference, are shown when responding in its first structural mode. The drag coefficients are calculated indirectly from the measured displacements. The dynamic response of the cylinder model used as the trailing one, but in a single circular cylinder arrangement has also been reported [15] and [16], together with the drag coefficients [17]), which appear compared with the results in this paper.

\section{EXPERIMENT DETAILS}

The set up in Figures 1 and 2 illustrates an aluminium supporting structure which was used to place the cylinder models in the water flume at the department of Aeronautics of Imperial College London.

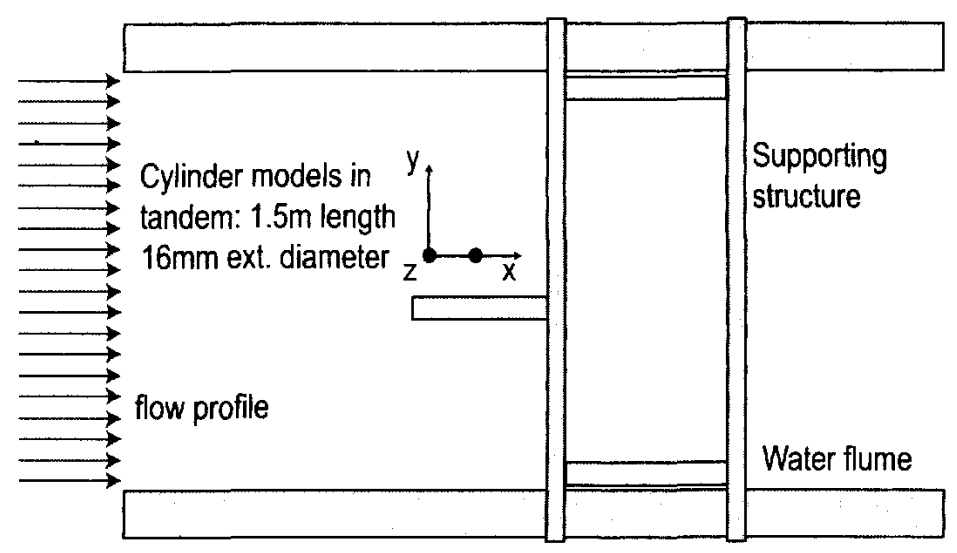

Figure 1. Top view of the experimental set up.

The models were attached to the structure through universal joints at both ends and the structure allowed changes in the centre to centre separation between the models $(S)$, as well as changes in the applied top tension $\left(T_{t}\right)$. As in previous experiments [8], only the lower 40 or $45 \%$ of the model was submerged in the flume. In that experiments [8] it was seen how that water coverage was enough to excite modes up to the $8^{\text {th }}$ cross-flow and the $14^{\text {th }}$ in-line. In the present work excitations only in the first or the second mode were expected. The design of the models and the instrumentation was based on previous experiments [8], and they were manufactured at the Universitat Rovira i Virgili in Tarragona, Spain. They were both made of a $1.5 \mathrm{~m}$ long aluminium skeleton structure with an internal core diameter of $6 \mathrm{~mm}$. In order to allow space for the instrumentation cables without increasing the flexural stiffness of the model, $15 \mathrm{~mm}$ diameter aluminium diaphragms with holes on them, were fixed to the core by means of cyanoacrylate glue. An external PVC cover was used 


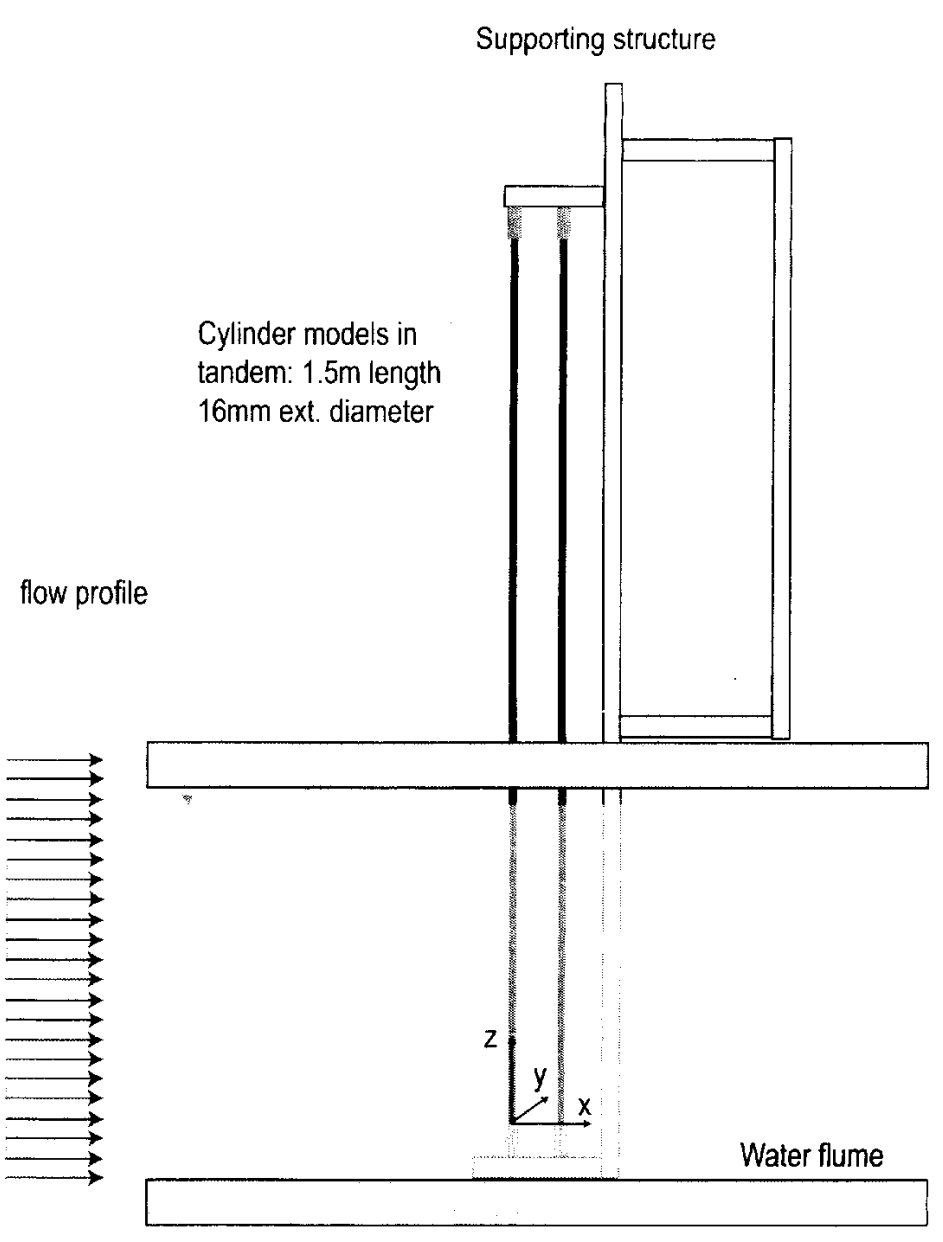

Figure 2. Side view of the experimental set up.

so the external diameter of the model ended up being $16 \mathrm{~mm}$, it also provided protection for the strain gauge instrumentation as water was not allowed inside the model. Figure 3 shows a detail of the finished instrumented model.

Only one of the two models was instrumented with strain gauges, and this was always used as the downstream cylinder in the tandem configuration. Five measurement stations were distributed along the length of this model, allowing the acquisition of cross-flow and in-line curvatures at those points, separated $300 \mathrm{~mm}$ between them, starting at $150 \mathrm{~mm}$ from both ends. A double spatial integration of the curvatures yielded to the displacements,

$$
\begin{aligned}
& c_{x}(z, t)=\frac{\partial^{2} x(z, t)}{\partial z^{2}} \\
& c_{y}(z, t)=\frac{\partial^{2} y(z, t)}{\partial z^{2}}
\end{aligned}
$$

where $x$ are $y$ are the in-line and transverse motions respec-

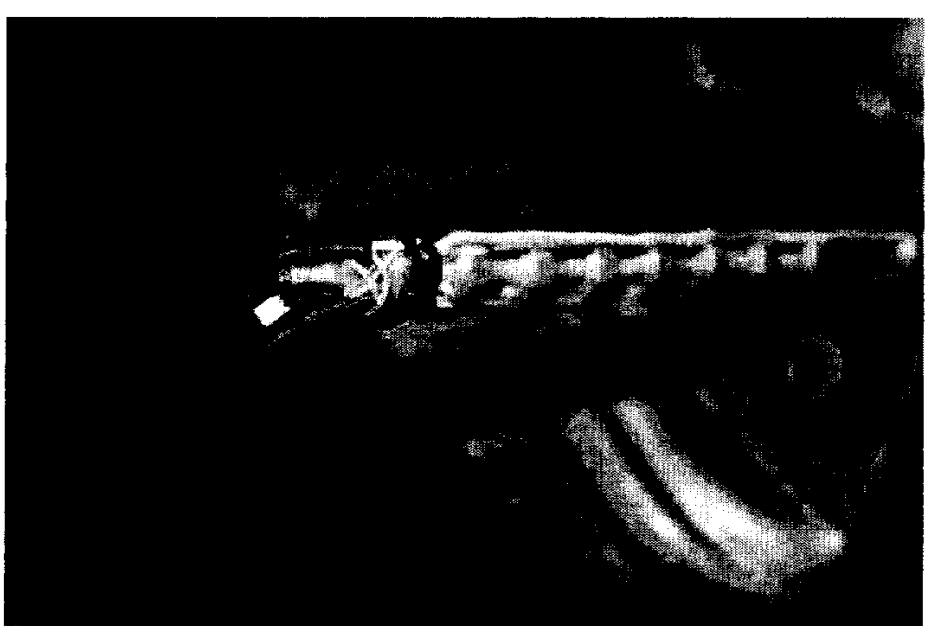

Figure 3. Detail of the cylinder model design.

tively, which can be calculated as follows,

$$
\begin{aligned}
& x(z, t)=\int_{0}^{L} \int_{o}^{L} \frac{\partial^{2} x(z, t)}{\partial z^{2}} d z d z \\
& y(z, t)=\int_{0}^{L} \int_{0}^{L} \frac{\partial^{2} y(z, t)}{\partial z^{2}} d z d z
\end{aligned}
$$

The boundary conditions for these expressions are those of a pin ended beam that has zero displacements and zero curvatures at the ends,

$$
\begin{array}{rlr}
x(z, t)=y(z, t)=0 & z=0, L & \forall t \\
\frac{\partial^{2} x(z, t)}{\partial z^{2}}=\frac{\partial^{2} y(z, t)}{\partial z^{2}}=0 & z=0, L & \forall t
\end{array}
$$

The upstream cylinder leading the tandem, did not have strain gauge instrumentation embedded on it, but the time series of the cross-flow displacement at its mid length was measured using a laser sensor. The fact that this cylinder did not have strain gauge instrumentation on it, made it slightly lighter because of the absence of cables.

Non-dimensional centre to centre separations $(S / D)$ of 1.5 , $2,2.5,3,3.5$ and 4 diameters were configured in different sets of experiments. At each one of these separations, three different top tensions $\left(T_{t}\right)$ of 15,60 and $110 \mathrm{~N}$ were configured, and for each tension the current in the water flume was varied up to $0.7 \mathrm{~m} / \mathrm{s}$ with increments of $0.1 \mathrm{~m} / \mathrm{s}$. Reynolds numbers of up to about 12000 , always in the subcritical regime, and reduced velocities based on the fundamental natural frequency of the downstream cylinder in water (equation 4) up to 16 were achieved. Table 1 


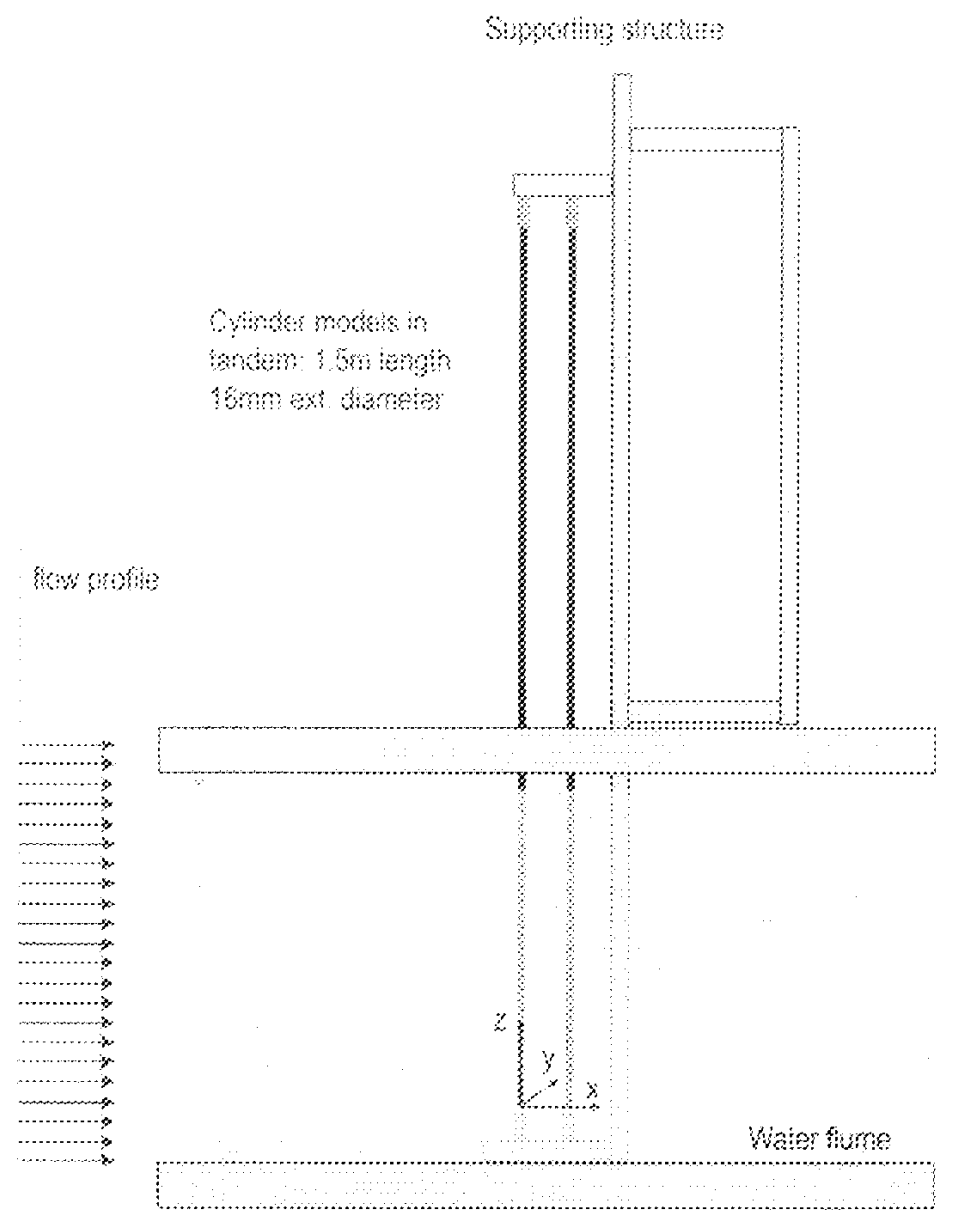

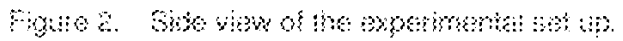

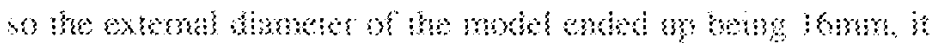

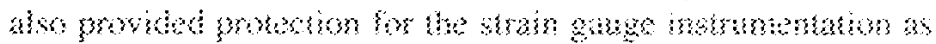

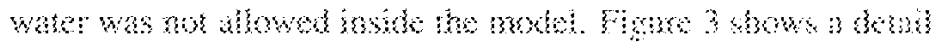

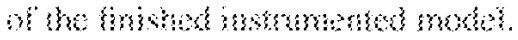

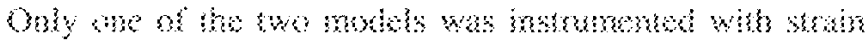

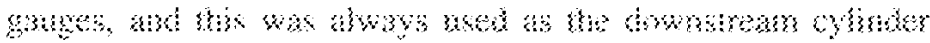

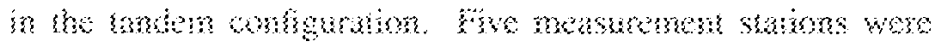

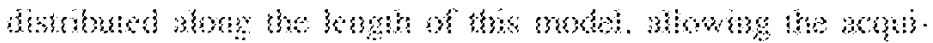

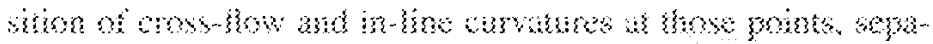

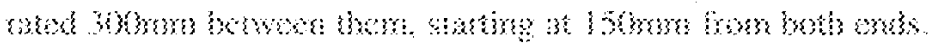

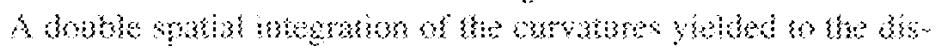
pesemens,

$$
\begin{aligned}
& (x)=\frac{b^{2} \times(x)}{n^{2}}
\end{aligned}
$$

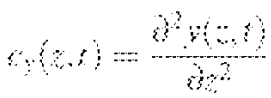

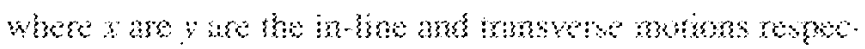

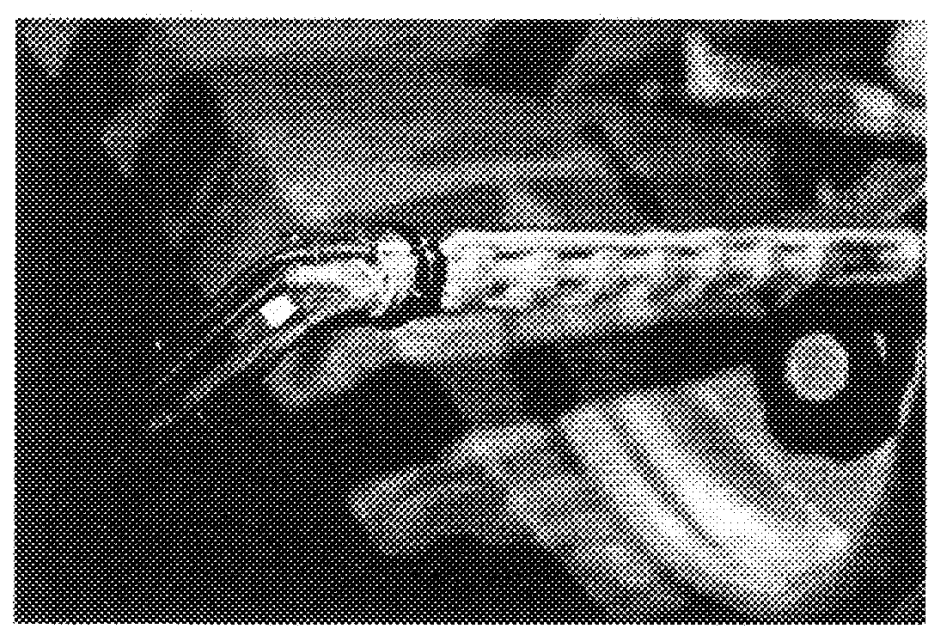

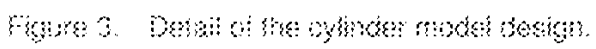

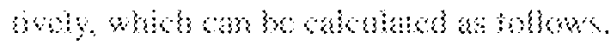

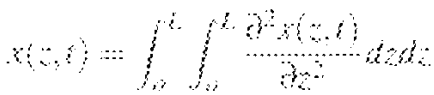

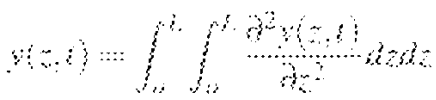

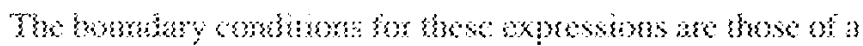

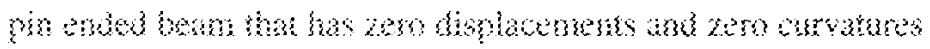
at he cols.

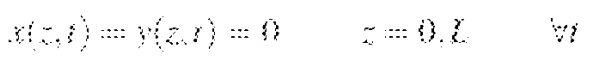

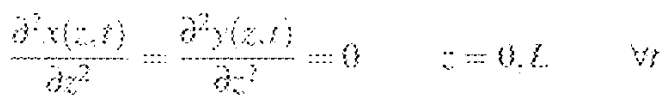

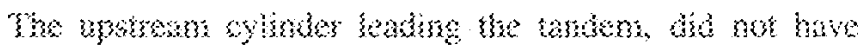

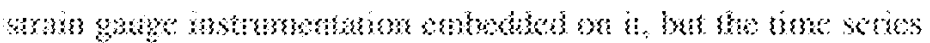

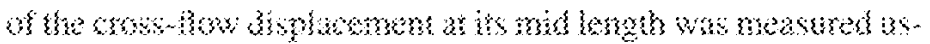

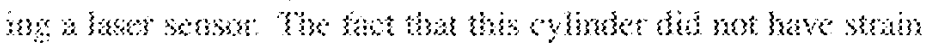

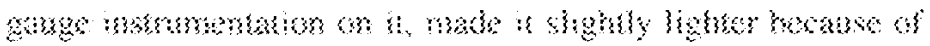
the nitsene of cobles.

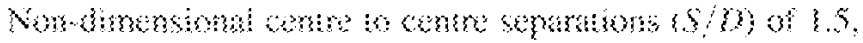

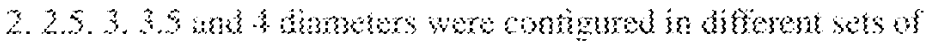

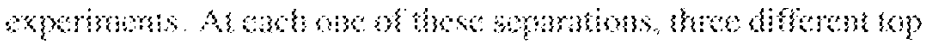

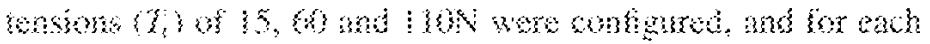

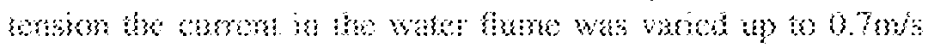

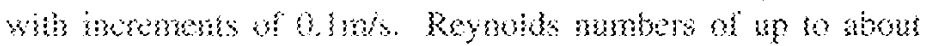

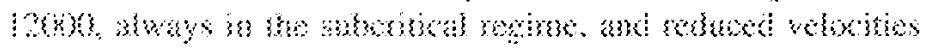

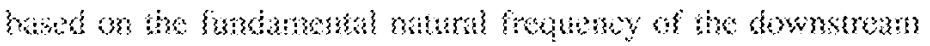

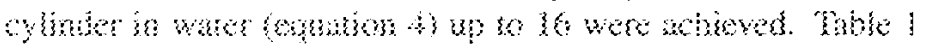


Table 1. NUMBER OF RUNS CONDUCTED FOR EACH TOP TENSION AND FOR EACH CENTRE TO CENTRE SEPARATION OF THE MODELS.

\begin{tabular}{lccccccc}
\hline \multicolumn{1}{c}{ Initial top tension $\left(T_{t}\right)$} & \multicolumn{6}{c}{ Centre to centre distance $(S / D)$} \\
$\mathrm{N}$ & Symbol & 1.5 & 2 & 2.5 & 3 & 3.5 & 4 \\
\hline 15 & $\square$ & 11 & 26 & 25 & 24 & 25 & 23 \\
60 & $\diamond$ & 12 & 24 & 22 & 25 & 22 & 20 \\
110 & $\circ$ & 21 & 24 & 19 & 23 & 20 & 23 \\
\hline \multicolumn{2}{l}{ Total number of runs } & 44 & 74 & 66 & 72 & 67 & 66 \\
\hline
\end{tabular}

shows a summary with the number of tests conducted for each testing situation.

$$
V_{1}=\frac{V}{f_{1} D}
$$

The fundamental natural frequency $\left(f_{1}\right)$ of the models was obtained in modal tests but also calculated with a finite element code which solves the eigenvalue problem of an Euler-Bernoulli beam [18] and [19]. For the instrumented model, it varied from about 3 to slightly over $7 \mathrm{~Hz}$, depending on the applied top tension, whilst it was about 3.2 to $8 \mathrm{~Hz}$ for the model without instrumentation. Structural modal damping ratios were obtained from decay tests in air after the model was forced to vibrate at a constant amplitude in a certain mode, and then the forcing was stopped. Damping ratios were obtained by analysing the exponential decay of amplitudes for each top tension tested. The values for both models as a percentage of critical damping were very similar and fell inside the range from $1.45 \%$ to $1.95 \%$, giving a mean value $\xi=1.77$. The main experimental details and the mechanical characteristics of both models appear on Table 2.

A typical in-line response of the trailing cylinder in one of the tests appears in Figure 4. All runs consisted of 90 seconds and they were initiated from still water as seen in the figure, in which the oscillations start approximately 10 seconds after the beginning of the run. The cross-flow response is not shown as it is not relevant for the calculation of the drag coefficients. The run was conducted with 4 diameters of separation between the models, a top tension of $110 \mathrm{~N}$ and a flow speed of $0.65 \mathrm{~m} / \mathrm{s}$. The reduced velocity was 5.45 and the Reynolds number was about 9100. The first column of plots shows the whole time series of the instantaneous in-line displacements at each one of the measurement stations. The second column shows only two seconds of these displacements enlarged and the last column shows their respective spectra. The data in general shows strong modulation around the mean value caused by the mean drag. The enlarged
Table 2. MAIN PARAMETERS OF THE EXPERIMENT, $\dagger$ REFERS TO UPSTREAM CYLINDER

\begin{tabular}{ccc}
\hline \multicolumn{3}{c}{ Experiment parameters } \\
\hline$D$ & $\mathrm{~m}$ & 0.016 \\
$L$ & $\mathrm{~m}$ & 1.5 \\
$L / D$ & - & 93.75 \\
$L_{s}$ & $\mathrm{~m}$ & 0.585 \\
$E I$ & $\mathrm{Nm}{ }^{2}$ & 6.04 \\
$E A$ & $\mathrm{MN}$ & 1.84 \\
$T_{t}$ & $\mathrm{~N}$ & $<110$ \\
$V$ & $\mathrm{~m} / \mathrm{s}$ & $<0.75$ \\
$S / D$ & - & $<4$ \\
$R e$ & - & $1200-12000$ \\
$m$ & $\mathrm{~kg} / \mathrm{m}$ & $0.362 \mid 0.293^{\dagger}$ \\
$w_{s}$ & $\mathrm{~N} / \mathrm{m}$ & $2.7 \mid 2.2^{\dagger}$ \\
$m^{*}$ & - & $1.8 \mid 1.45^{\dagger}$ \\
$\left(m^{*}+C_{A}\right) \zeta$ & - & $0.049 \mid 0.043^{\dagger}$ \\
\hline
\end{tabular}

data and the spectra indicate a very clear dominant frequency.
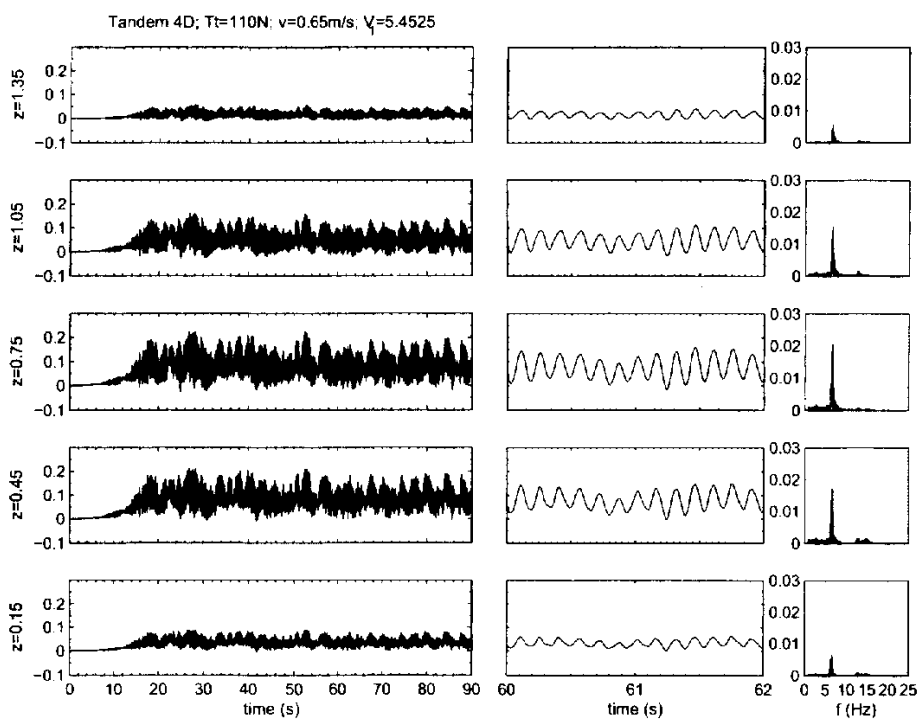

Figure 4. Instantaneous non-dimensional in-line displacements. Run with $S / D=4, V=0.65 \mathrm{~m} / \mathrm{s}$ and $T t=110 \mathrm{~N}$. First column shows the whole time series, the second shows an enlargement of 2 seconds inside the run and the last column depicts the spectra of each signal. 


\section{CALCULATION OF THE DRAG COEFFICIENTS}

It was observed that the variations in flexural stiffness for the different initial applied top tension was not negligible and hence these changes have been taken into account. The tension was not monitored during each run, but the difference between the initial and the mean tension during the experiment was expected to be small. compared to the differences between each one of the initial imposed tensions $(15.60$ and $110 \mathrm{~N})$, as it was observed in previous experiments [8]. By measuring the deflection under known point loads applied to the mid point of the model, and assuming the resulting deformed shape to be sinusoidal, one can derive from the Euler-Bernoulli beam theory, an equation that gives an estimate for a modified flexural stiffness $\left(E I^{*}\right)$ as a function of the top tension, the load and the deformation measured,

$$
E I^{*}=\frac{-L^{2}}{\pi^{2}}\left[\frac{W L^{2}}{\pi^{2} x_{M}}+T_{i}\right]
$$

where $W$ is the applied point load and $x_{M}$ is the maximum deformation at the mid point.

The drag coefficients have been obtained by using a finite element code described in [18] and [19]. This method was found to be successful in calculating force coefficients from deflections. Comparisons between computed and independently measured drag coefficients were in close agreement [19]. As this work is concerned with the mean drag coefficients acting on the cylinder model, the time evolution of the forces has not been computed. For the sake of simplicity, it has been assumed that the mean deflection calculated from the instantaneous measured inline displacements, can be obtained by applying a uniform force distribution $f_{x}(z)$ to the submerged part of the model $\left(L_{s}\right)$. The integration of this force distribution along the length of the model gives the total force acting on the cylinder $F_{X}$,

$$
F_{x}=\int_{0}^{L} f_{x}(z) d z
$$

The $C_{d}$ can then be obtained,

$$
C_{d}=\frac{F_{x}}{\frac{1}{2} \rho V^{2} L_{s}}
$$

An iterative process in which an initial value for the $C_{d}$, equal to the expected drag coefficient for a stationary cylinder at the same $R e$, has been used to construct a uniform force distribution along the submerged part of the model as in Figure 5(b).
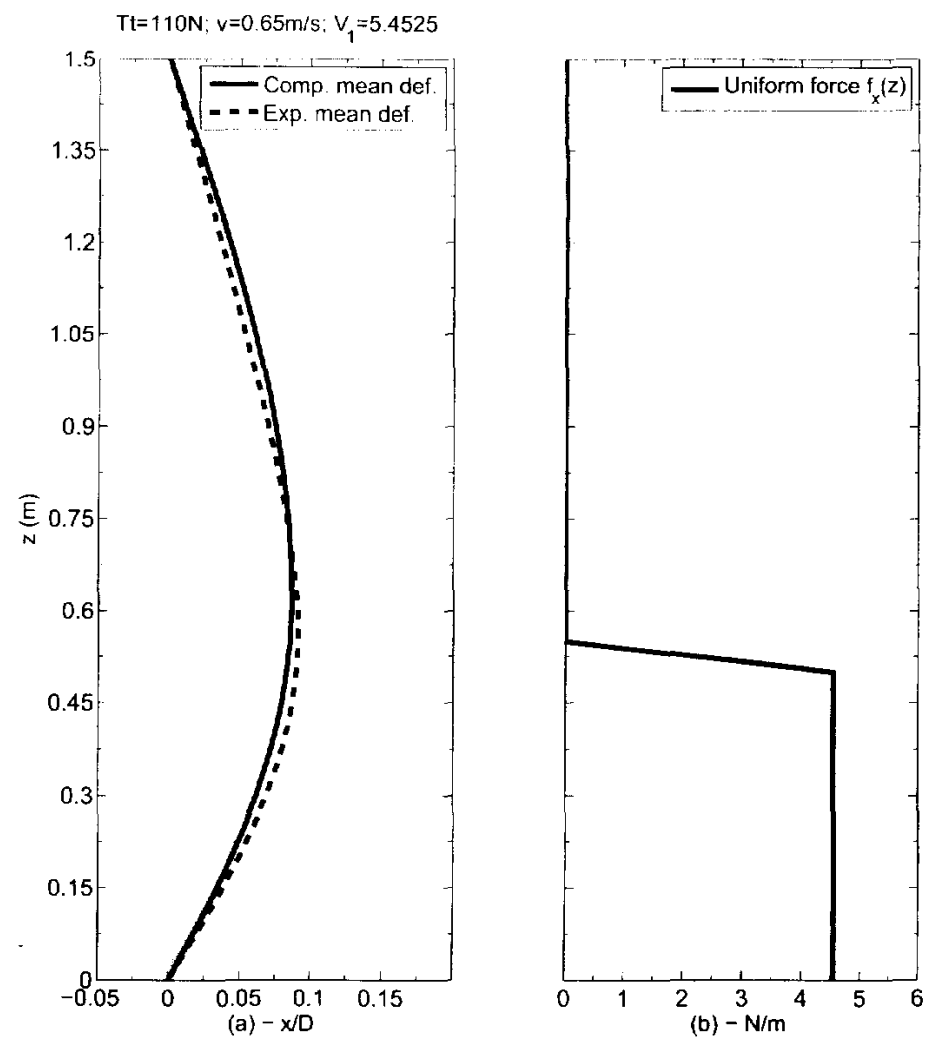

Figure 5. Example of mean deflection used for drag coefficient calculation. The plot in the left hand side shows the in-line instantaneous deflections every $1 / 10^{\text {th }}$ of a second with solid grey lines. Experimental (dashed line) and computed (solid line) mean deflections are also shown in the plot. The right hand side shows the computed uniform force distribution that produces the computed deflection shown in the left hand side.

By inputting that distribution to the finite element code, the corresponding deflection can be computed and compared to the experimental one obtained from the experiments. In Figure 5(a) the computed mean deflection is shown with a solid line and the experimental one with a dashed line for the same example run that appeared in Figure 4. Also shown are the instantaneous deflections every one tenth of a second from the $65^{\text {th }}$ to the $70^{\text {th }}$ second. After each iteration, if the deflections are different, the drag coefficient is updated. When the two deflections match each other, it means that the uniform force distribution based on the recently updated drag coefficient, would produce the same deflection on the model as the produced by the flow in the experiment. The matching criteria used, stops the updating process of the $C_{d}$ when the area enclosed by the deflections of both curves and the $z$ axis, differ less than a certain percentage value which was set to $0.1 \%$. The accuracy of the results presented here, is largely dependent on the accuracy of the displacement measurements, as they are the input to the FEM code. In order to verify them, an initial set of verification tests was carried out with only 

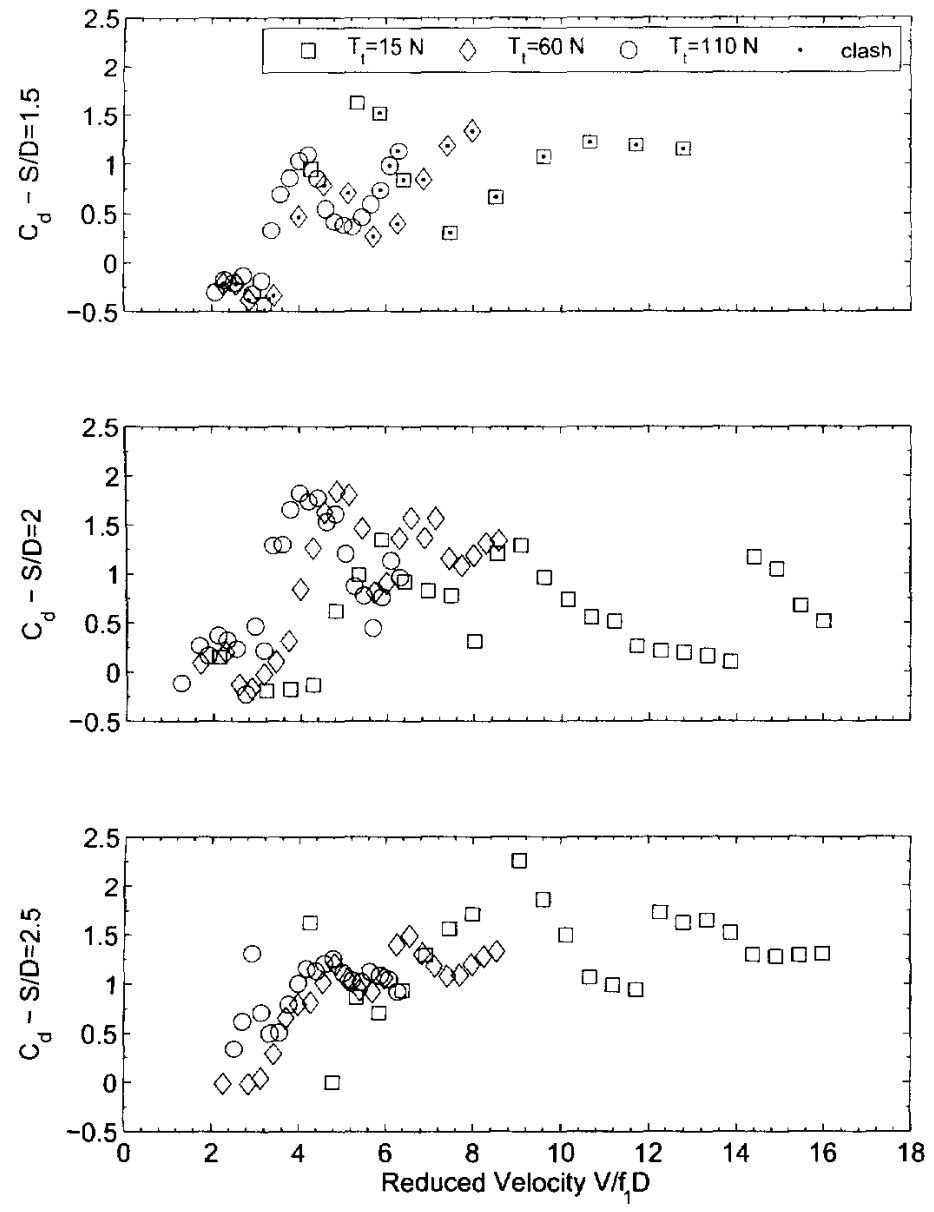

Figure 6. Drag coefficients acting on the downstream cylinder in the tandem arrangement with a centre to centre separation of 1.5, 2 and 2.5 diameters, as a function of the reduced velocity $V_{1}$. The data appears grouped inside each plot for the three sets of top tension tested.

the instrumented model [16]. The displacements given by the strain gauge stations were simultaneously measured with a laser sensor, allowing the comparison of the two independent signals. Very good agreement was seen in all the stations.

\section{RESULTS}

The results of applying the method described in the previous section is shown in Figures 6 and 7. The mean drag coefficients appear grouped in different plots for each centre to centre separation and with different symbols for each applied top tension.

For centre to centre distances up to 3 diameters and reduced velocities smaller than 4 , the downstream cylinder model experiences very low drag coefficients, going down to negative in some cases. With gap distances up to 2.5 diameters, there is a rapid increase in the drag coefficient from negative to values around 1 , followed by a sudden drop to values of about 0.5 and an in-
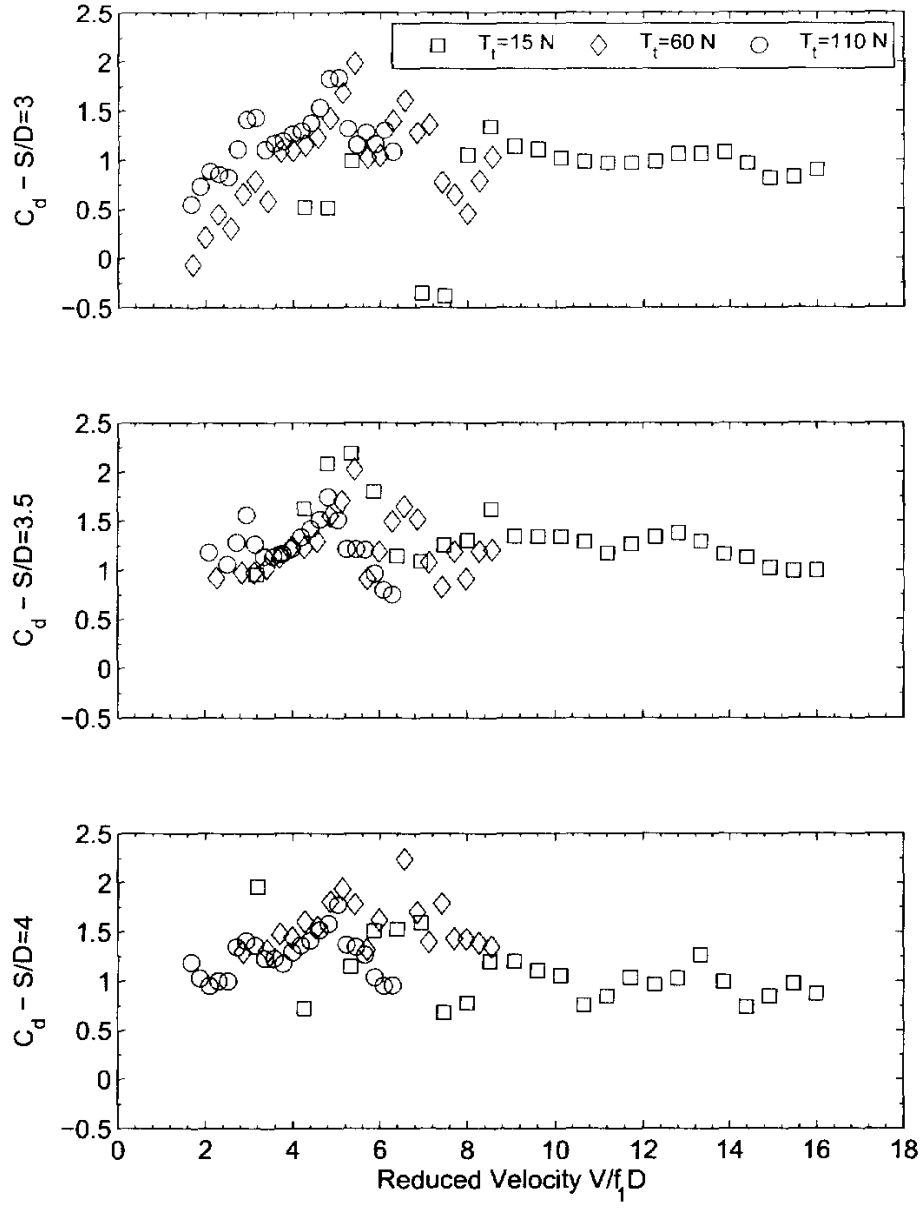

Figure 7. Drag coefficients acting on the downstream cylinder in the tandem arrangement with a centre to centre separation of 3, 3.5 and 4 diameters, as a function of the reduced velocity $V_{1}$. The data appears grouped inside each plot for the three sets of top tension tested.

crease to values slightly over 1 . The black dots in the upper plot of Figure 6, for $S / D=1.5$, indicate clashing between the models. Clashing occurred at the highest speeds with the lowest top tensions and also at low speeds because of the suction created in the near wake.

For $S / D>2$ and reduced velocities higher than 4 , the vast majority of the points is situated between 1 and 2 . Peak values reach 2.25 at reduced velocities near 6 . It is seen here how the amplification of the drag coefficient with the vibration amplitude does happen as well in circular cylinders with wake interference, as the largest motions on the downstream cylinder take place for reduced velocities in the range from 4 to 10 [15]. For reduced velocities smaller than 4 and small gap distances, the motion of the trailing cylinder was very small [15] and it is confined in the near wake of the upstream cylinder so negative $C_{d}$ s are observed.

Figure 8 shows the drag coefficients for all gap distances (the same ones as in Figures 6 and 7), together with the drag 


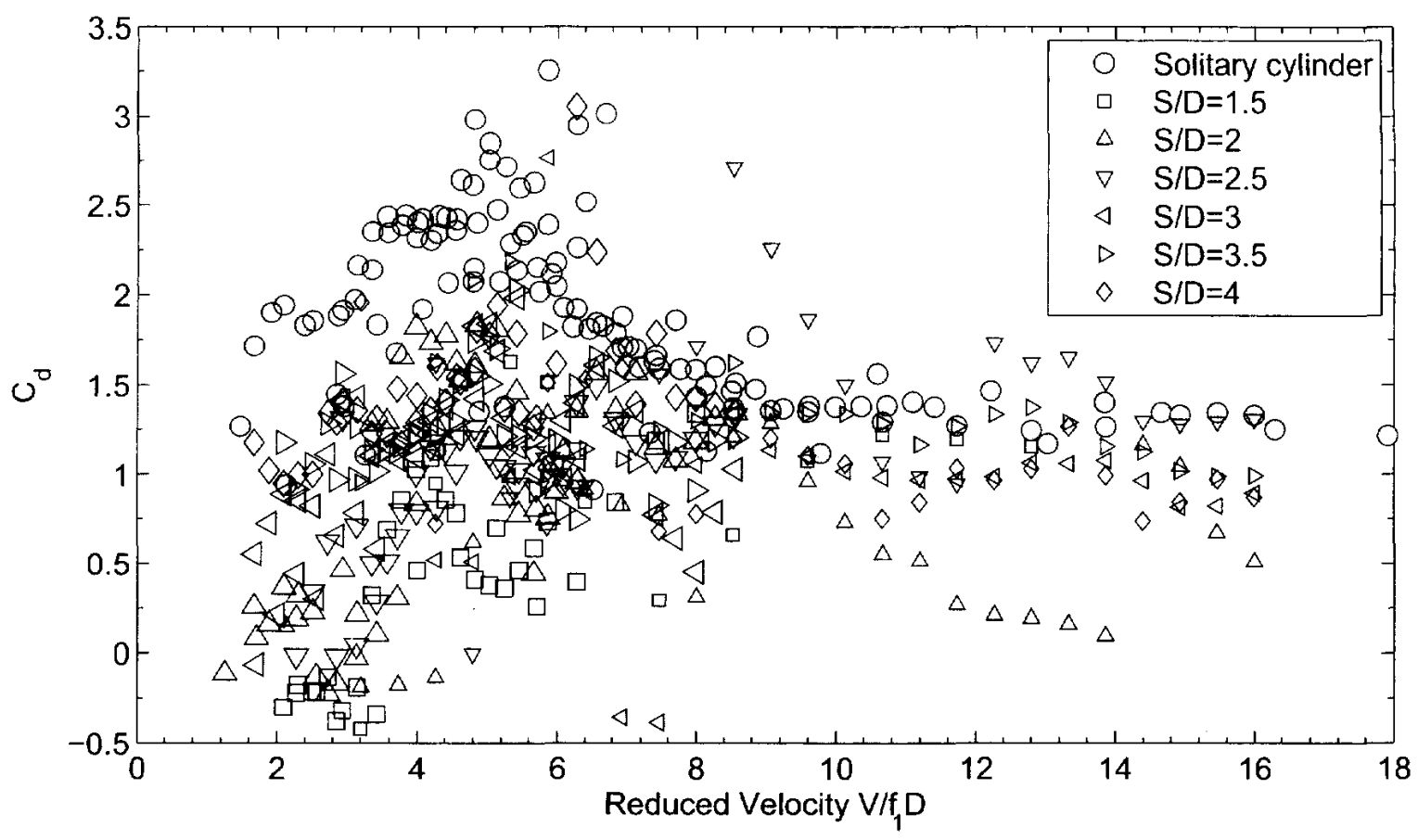

Figure 8. Comparison between the drag coefficients exhibited by the downstream cylinder in the tandem arrangement (hollow symbols) with those experienced by the solitary cylinder (solid symbols).

coefficients acting on the same model but in a single cylinder arrangement as presented in [17]. For reduced velocities smaller than 6 , the difference between the drag coefficients of the solitary cylinder and the cylinder with wake interference is much higher than at higher reduced velocities. At these low reduced velocities, the solitary cylinder exhibits $C_{d} \mathrm{~s}$ in the range from 1.75 to 3 whilst the drag coefficient for the downstream cylinder in the tandem has values oscillating between -0.5 and 2 . As the reduced velocity is increased, the $C_{d} \mathrm{~s}$ of the downstream cylinder in tandem become closer to those of the solitary one. independently of the centre to centre separation [15].

\section{CONCLUSIONS}

The drag coefficients acting on the downstream cylinder in an arrangement of two tandem cylinders have been presented. For reduced velocities smaller than 6 , there is a very strong dependence of the drag coefficients on the separation between the models. The larger the separation is, the larger the drag coefficients, tending to those found in single cylinder arrangements. Values of -0.5 are reached at the smallest separation distances and clashing is observed. For reduced velocities larger than 10 the dependence on the separation distance between the models becomes weaker, the values of the drag coefficient are between 1 and 1.5 , tending to those typical of solitary cylinders at the largest separations.
The amplification in drag due to the large amplitudes under lock-in conditions experienced by single cylinders, is also observed in vortex-induced vibrations with wake interference. The drag coefficients in the trailing cylinder of the tandem arrangement are large when the largest amplitude of vibrations are observed under lock-in conditions.

\section{ACKNOWLEDGMENT}

Special thanks are due to Prof. P.W. Bearman and the Department of Aeronautics of Imperial College London. Funding provided by Universitat Rovira i Virgili through grant 2006AIRE-03 is gratefully acknowledged. The author is a Marie Curie Postdoctoral Research Fellow funded by the European Commission (PIOF-GA-2008-219429).

\section{REFERENCES}

[1] Sarpkaya, T., 1979. "Vortex-induced oscillations, a selective review". Journal of Applied Mechanics, 46, pp. 24I258.

[2] Bearman, P. W., 1984. "Vortex shedding from oscillating bluff bodies". Annual Review of Fluid Mechanics, 16, pp. 195-222.

[3] Blevins, R. D., 1990. Flow-Induced Vibration, second ed. Van Nostrand Reinhold. 
[4] Parkinson, G., 1989. "Phenomena and modelling of flowinduced vibrations of bluff bodies". Progress in Aerospace Sciences, 26, pp. 169-224.

[5] Sarpkaya, T.. 2004. "A critical review of the intrinsic nature of vortex-induced vibrations". Journal of Fluids and Structures, 19, pp. 389-447.

[6] Williamson, C., and Govardhan, R., 2004. "Vortexinduced vibrations". Annual Review of Fluid Mechanics, 36, pp. 413-55.

[7] Chaplin, J. R., Bearman, P. W., Cheng, Y., Fontaine, E., Graham, J. M. R., Herfjord, K., Huera-Huarte. F. J., Isherwood, M., Lambrakos, K., Larsen, C. M., Meneghini, J. R., Moe, G., Pattenden, R. J., Triantafyllou, M. S., and Willden, R. H. J., 2005. "Blind predictions of laboratory measurements of vortex induced vibrations of a tension riser". Journal of Fluids and Structures, 21(1), pp. 25-40.

[8] Chaplin, J. R., Bearman, P. W., Huera-Huarte, F. J., and Pattenden, R., 2005. "Laboratory measurements of vortexinduced vibrations of a vertical tension riser in a stepped current". Journal of Fluids and Structures, 21(1), pp. 3-24.

[9] Vandiver, J. K., 1983. "Drag coefficients of long flexible cylinders". In Offshore Technology Conference, no. OTC 4490.

[10] de Wilde, J. J., and Huijsmans, R. H., 2004. "Laboratory investigation of long riser viv response". In Procedings of the 14th International Offshore and Polar Conference, pp. 511516.

[11] Bokaian, A., and Geoola, F., 1984. "Wake-induced galloping of two interfering circular cylinders". Journal of Fluid Mechanics, 146, pp. 383-415.

[12] Zdravkovich, M. M., 1985. "Flow induced oscillations of two interfering circular cylinders". Journal of Sound and Vibration, 101, pp. 511-521.

[13] Hover, F. S., and Triantafyllou, M. S., 2001. "Galloping response of a cylinder with upstream wake interference". Joumal of Fluids and Structures, 15, pp. 503-512.

[14] Assi, G. R. S., Meneghini, J. R., Aranha, J. A. P., Bearman, P. W., and Casaprima, E., 2006. "Experimental investigation of flow-induced vibration interference between two circular cylinders". Journal of Fluids and Structures, 22, pp. 819-827.

[15] Huera-Huarte, F. J., and Bearman, P. W., 2008. "Wake structures and dynamic reponse of one and two long flexible cylinders undergoing vortex-induced vibrations". In Procedings of the 9th International Conference on FlowInduced Vibrations.

[16] Huera-Huarte, F. J., and Bearman, P. W., submitted 2008. "Wake structures and vortex-induced vibrations of a long flexible cylinder - part 1: Dynamic response". Journal of Fluids and Structures.

[17] Huera-Huarte, F. J., and Bearman, P. W., submitted 2008. "Wake structures and vortex-induced vibrations of a long flexible cylinder - part 2: Drag coefficients and vortex modes". Journal of Fluids and Structures.

[18] Huera-Huarte, F. J., 2006. "Multi-mode vortex-induced vibrations of a flexible circular cylinder". PhD thesis, Imperial College London.

[19] Huera-Huarte, F. J., Bearman, P. W., and Chaplin, J. R., 2006. "On the force distribution along the axis of a flexible circular cylinder undergoing multi-mode vortex-induced vibrations". Iournal of Fluids and Structures, 22, pp. $897-$ 903. 